\title{
Interfacial Coordination Nanosheet Based on Non- \\ Conjugated Three-Arm Terpyridine: A Highly Color- Efficient Electrochromic Material to Converge Fast Switching with Long Optical Memory
}

\author{
Susmita Roy and Chanchal Chakraborty* \\ Department of Chemistry, Birla Institute of Technology and Science (BITS) Pilani, Hyderabad \\ Campus, Jawahar Nagar, Shameerpet Mandal, Hyderabad-500078, India \\ Email: Chanchal@hyderabad.bits-pilani.ac.in
}




\section{Content}

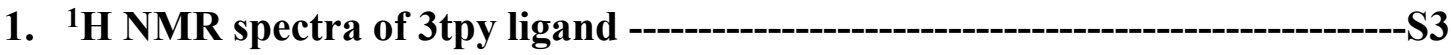

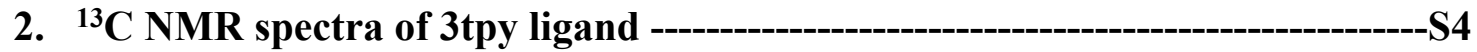

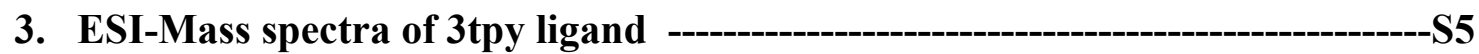

4. Optimization of the film thickness by varying reaction condition---------------S6

5. UV-Vis titration using 3tpy ligand as a function of $\mathrm{Fe}^{2+}$ ions --------------S7

6. XPS and TGA study of 3tpy-Fe film ---S8

7. UV-Vis spectra comparison of 3tpy-Fe polymers prepared in bulk and interfacial polymerizations -S9

8. Peak current vs. scan rate study of 3 tpy-Fe film in three electrode system-----S9

9. The EC properties of $3 t p y-F e$ film $(350 \mathrm{~nm})$ in three electrode system --------S10

10. Electrochromic and chronoamperometry study of 3tpy-Fe film based ECD -S11

11. EC memory as a function of thickness of 3 tpy-Fe film S13

12. Thickness measurement of polyFe and 3tpyC-Fe film S13 


\section{1. ${ }^{1} \mathrm{H}$ NMR spectra of 3tpy ligand}

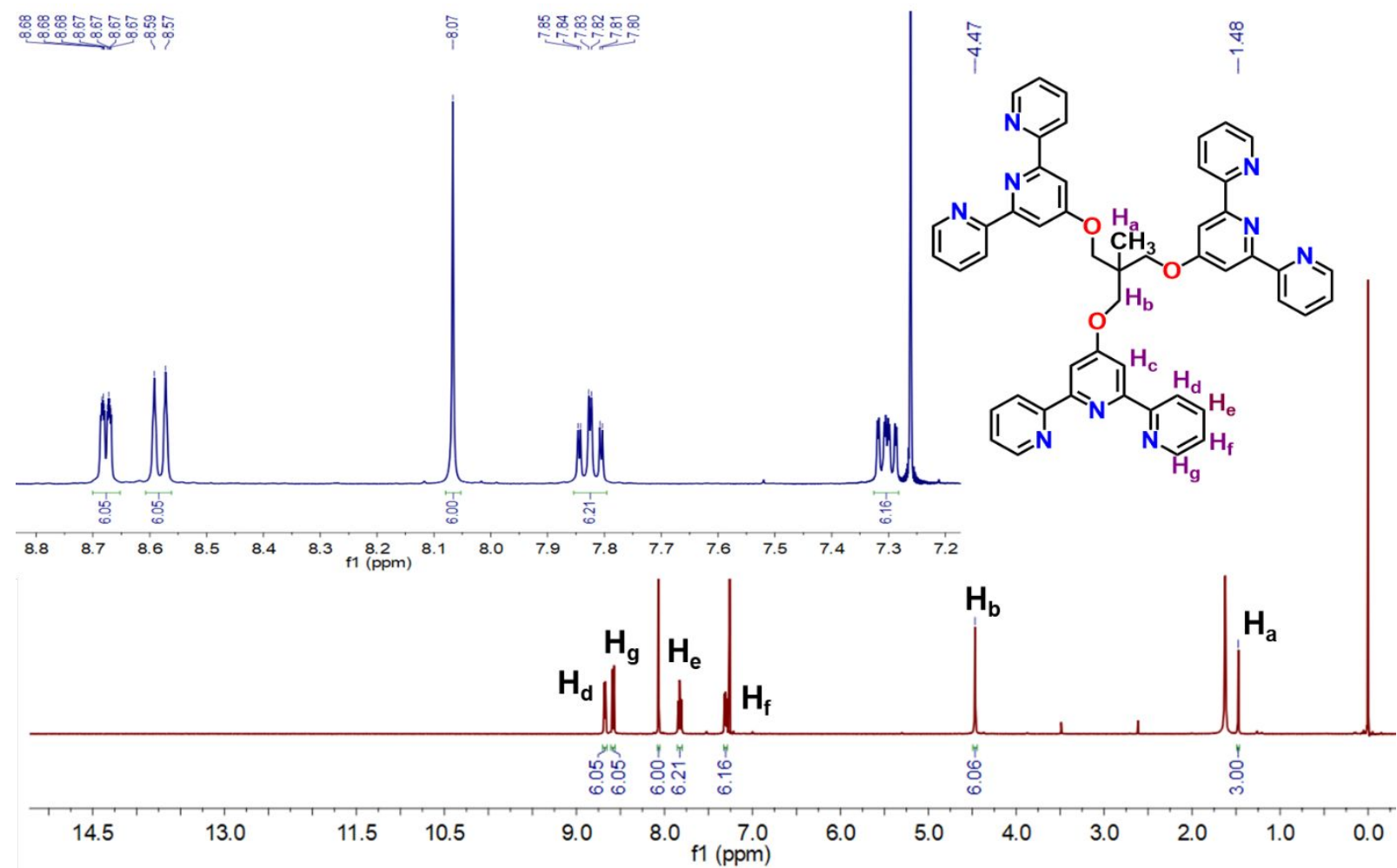

Figure S1. ${ }^{1} \mathrm{H}$ NMR spectra of 3 tpy ligand in $\mathrm{CDCl}_{3}$ solvent at room temperature. The chemical structure and the assignment of the different hydrogens are given. The aromatic zone is enlarged in the inset. 


\section{2. ${ }^{13} \mathrm{C}$ NMR spectra of 3tpy ligand}

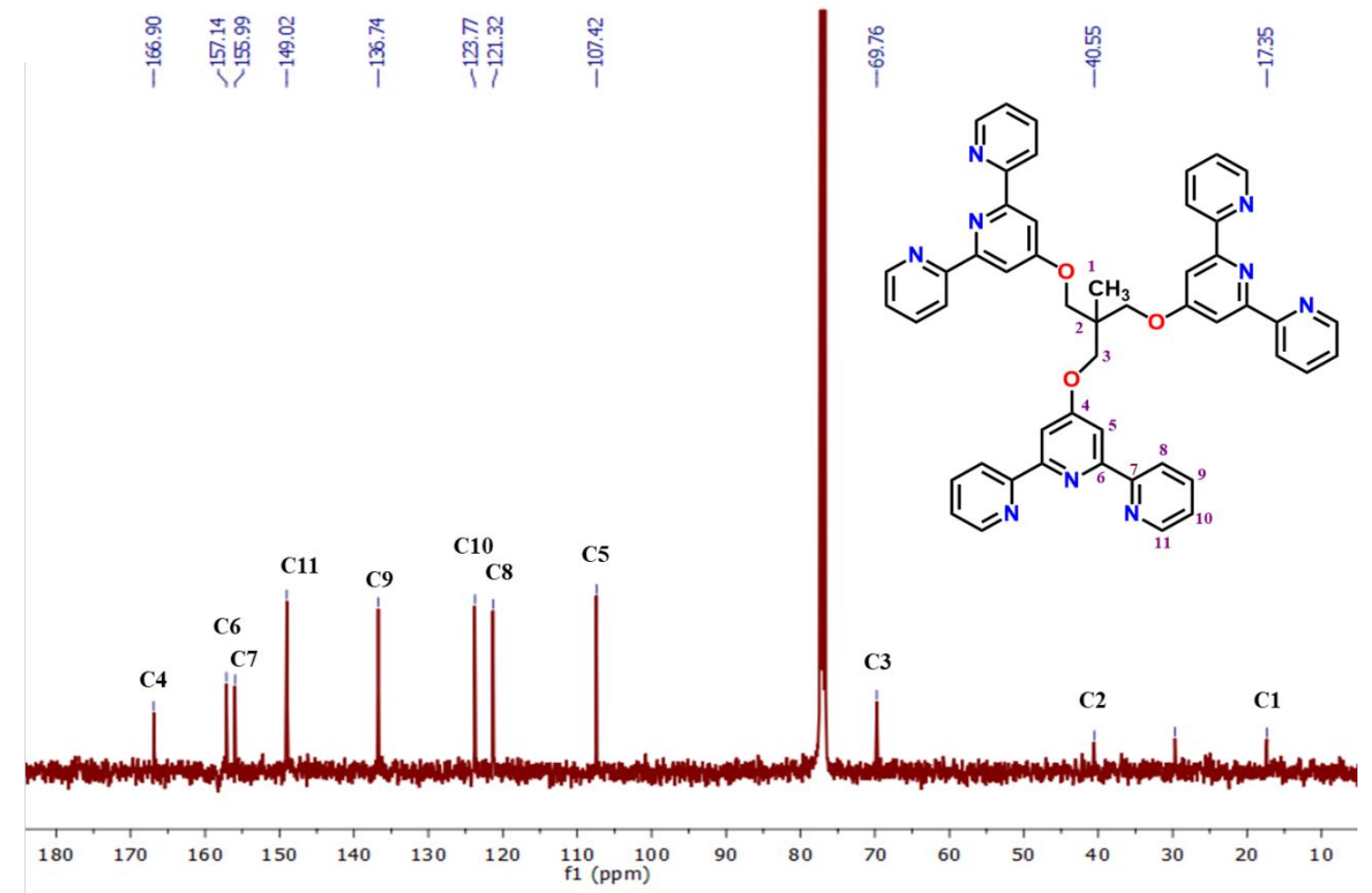

Figure S2. ${ }^{13} \mathrm{C}$ NMR spectra of 3tpy ligand in $\mathrm{CDCl}_{3}$ solvent at room temperature. The chemical structure and the assignment of the different carbons are given. 


\section{ESI-Mass spectra of 3tpy ligand}

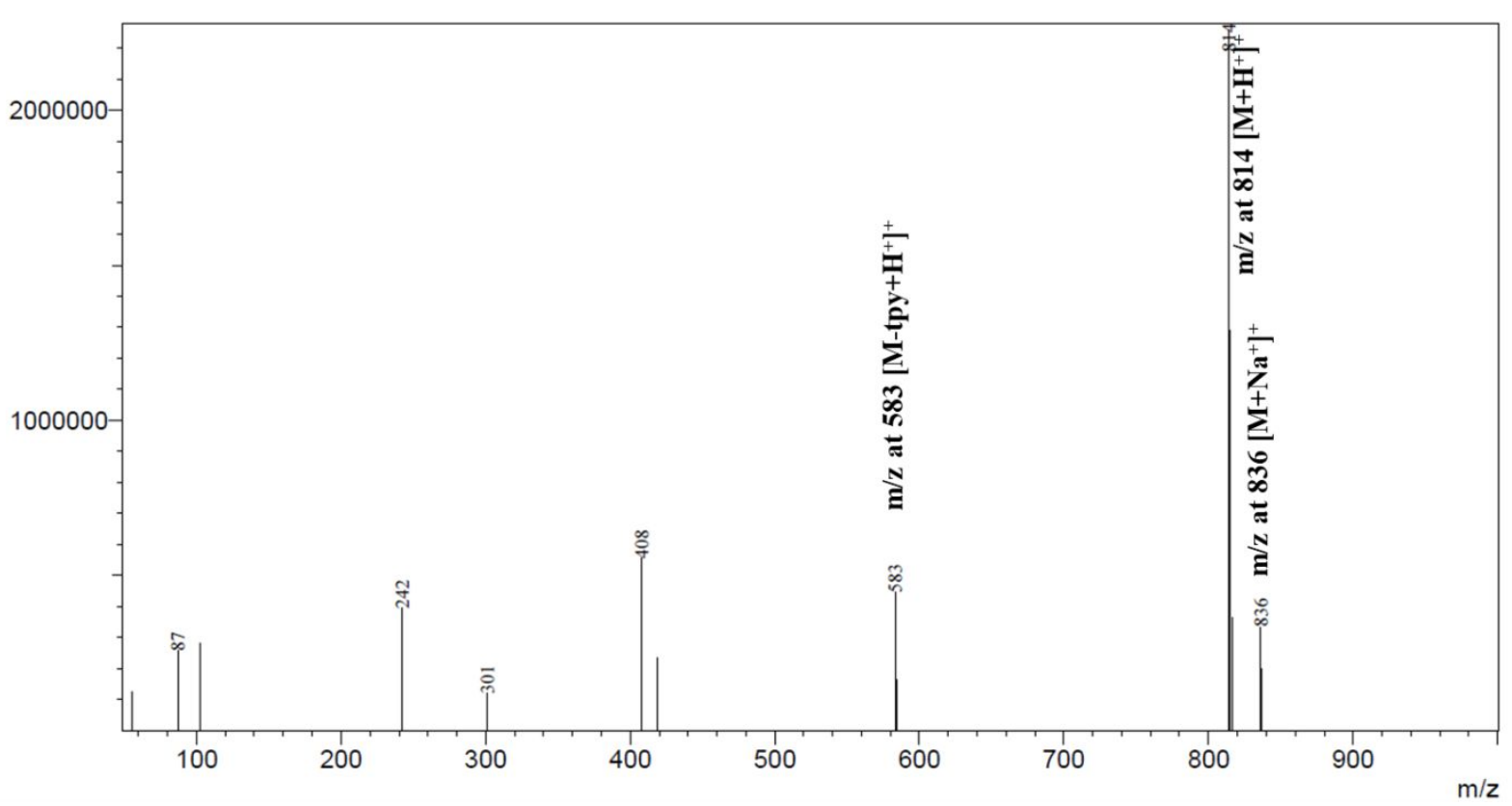

Figure S3. ESI-Mass spectra of 3tpy ligand. The important $\mathrm{m} / \mathrm{z}$ values are assigned in the picture. 
4. Optimization of the film thickness by varying reaction conditions
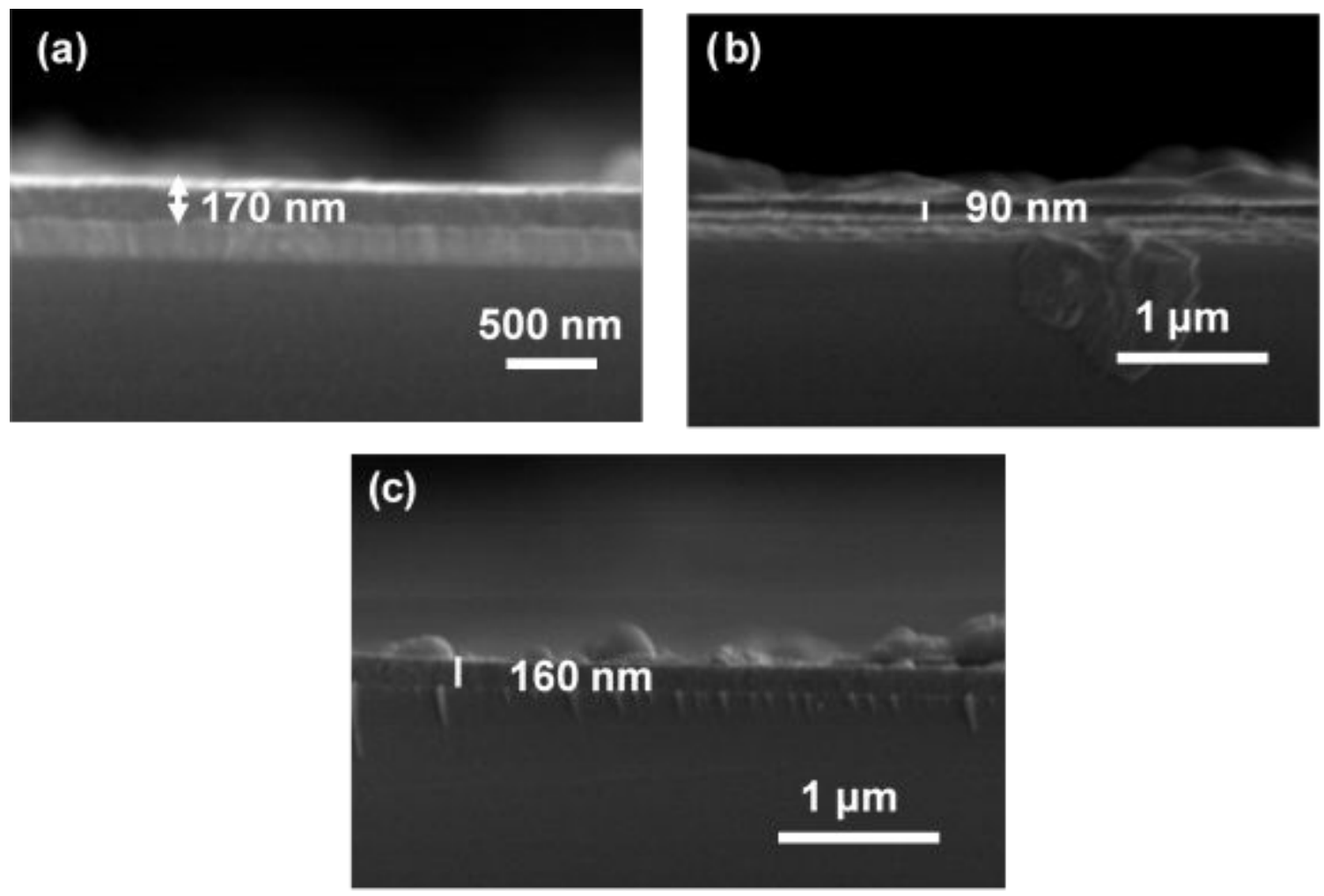

Figure S4. Thickness measurement through cross-sectional SEM study of 3tpy-Fe film using

(a) $50 \mathrm{mM} \mathrm{Fe}^{2+}$ ion with $0.1 \mathrm{mM} 3$ tpy for $36 \mathrm{~h}$, (b) $50 \mathrm{mM} \mathrm{Fe}^{2+}$ ion with $0.1 \mathrm{mM} 3$ tpy for 24 h, and (c) $25 \mathrm{mM} \mathrm{Fe}^{2+}$ ion with $0.1 \mathrm{mM} 3$ tpy for $48 \mathrm{~h}$.

Table S1. Summary of the thickness and optical contrast of the films formed by varying the conditions.

\begin{tabular}{|l|l|l|l|l|l|}
\hline Entry & $\begin{array}{l}\text { Concentration } \\
\text { of 3tpy }(\mathbf{m M})\end{array}$ & $\begin{array}{l}\text { Concentration } \\
\text { of } \mathbf{F e}^{\mathbf{2}} \text { ions } \\
\mathbf{( m M )}\end{array}$ & $\begin{array}{l}\text { Reaction } \\
\text { time (h) }\end{array}$ & $\begin{array}{l}\text { Average film } \\
\text { thickness (nm) }\end{array}$ & $\begin{array}{l}\text { Optical } \\
\text { contrast } \boldsymbol{\Delta T} \\
\mathbf{( \% )}\end{array}$ \\
\hline 1 & $0.1 \mathrm{mM}$ & $50 \mathrm{mM}$ & $48 \mathrm{~h}$ & $\sim 350 \mathrm{~nm}$ & $53 \%$ \\
\hline 2 & $0.1 \mathrm{mM}$ & $50 \mathrm{mM}$ & $36 \mathrm{~h}$ & $\sim 170 \mathrm{~nm}$ & $37 \%$ \\
\hline 3 & $0.1 \mathrm{mM}$ & $50 \mathrm{mM}$ & $24 \mathrm{~h}$ & $\sim 90 \mathrm{~nm}$ & $29 \%$ \\
\hline 4 & $0.1 \mathrm{mM}$ & $25 \mathrm{mM}$ & $48 \mathrm{~h}$ & $\sim 160 \mathrm{~nm}$ & $35 \%$ \\
\hline
\end{tabular}

\# $\Delta T$ is optical contrast (transmittance difference between bleached and colored state during solid state EC measurement). 
5. UV-Vis titration using 3 tpy ligand as a function of $\mathrm{Fe}^{2+}$ ions
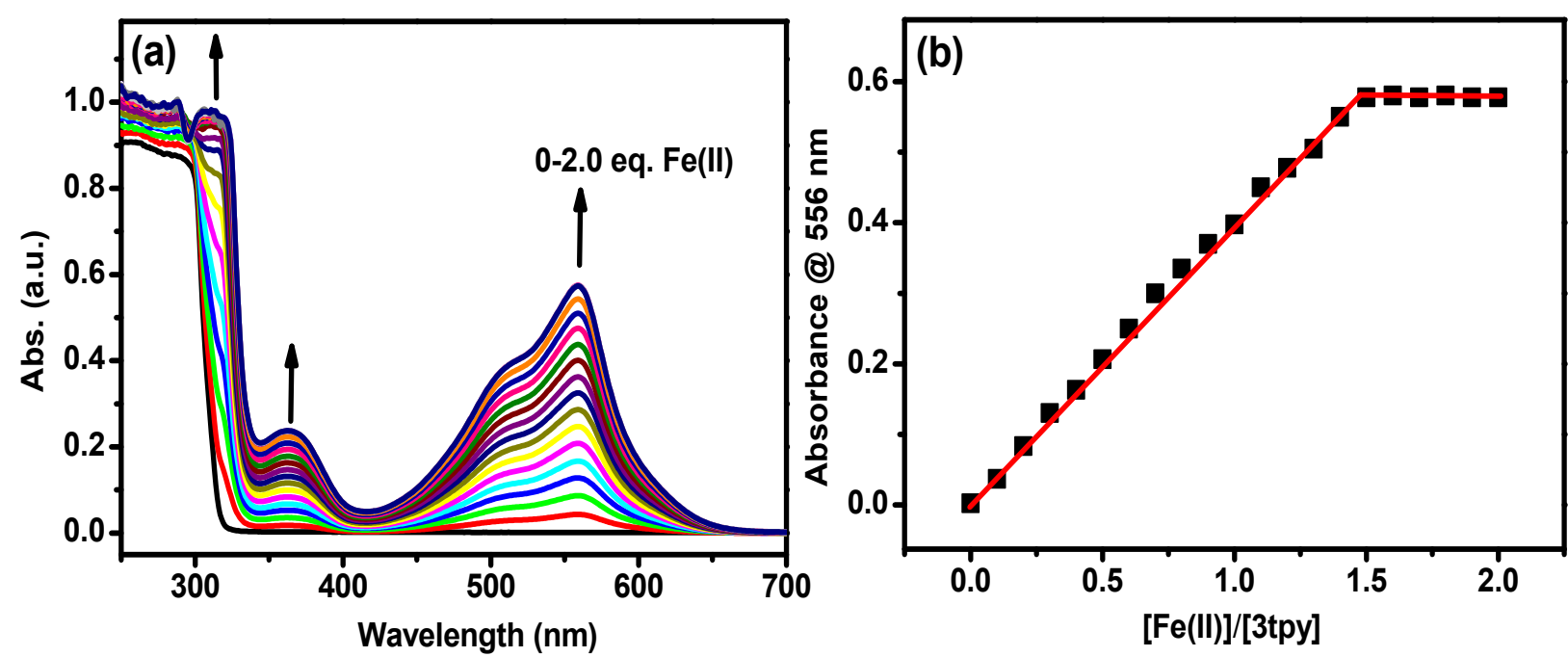

Figure S5. (a) UV-vis spectral change of a methanol solution of 3tpy during titration with $\mathrm{Fe}\left(\mathrm{BF}_{4}\right)_{2}$ at room temperature. (b) The absorption change at $556 \mathrm{~nm}$ as a function of the $\left[\mathrm{Fe}\left(\mathrm{BF}_{4}\right)_{2}\right] /[$ 3tpy] ratio. 
6. XPS and TGA study of 3tpy-Fe film
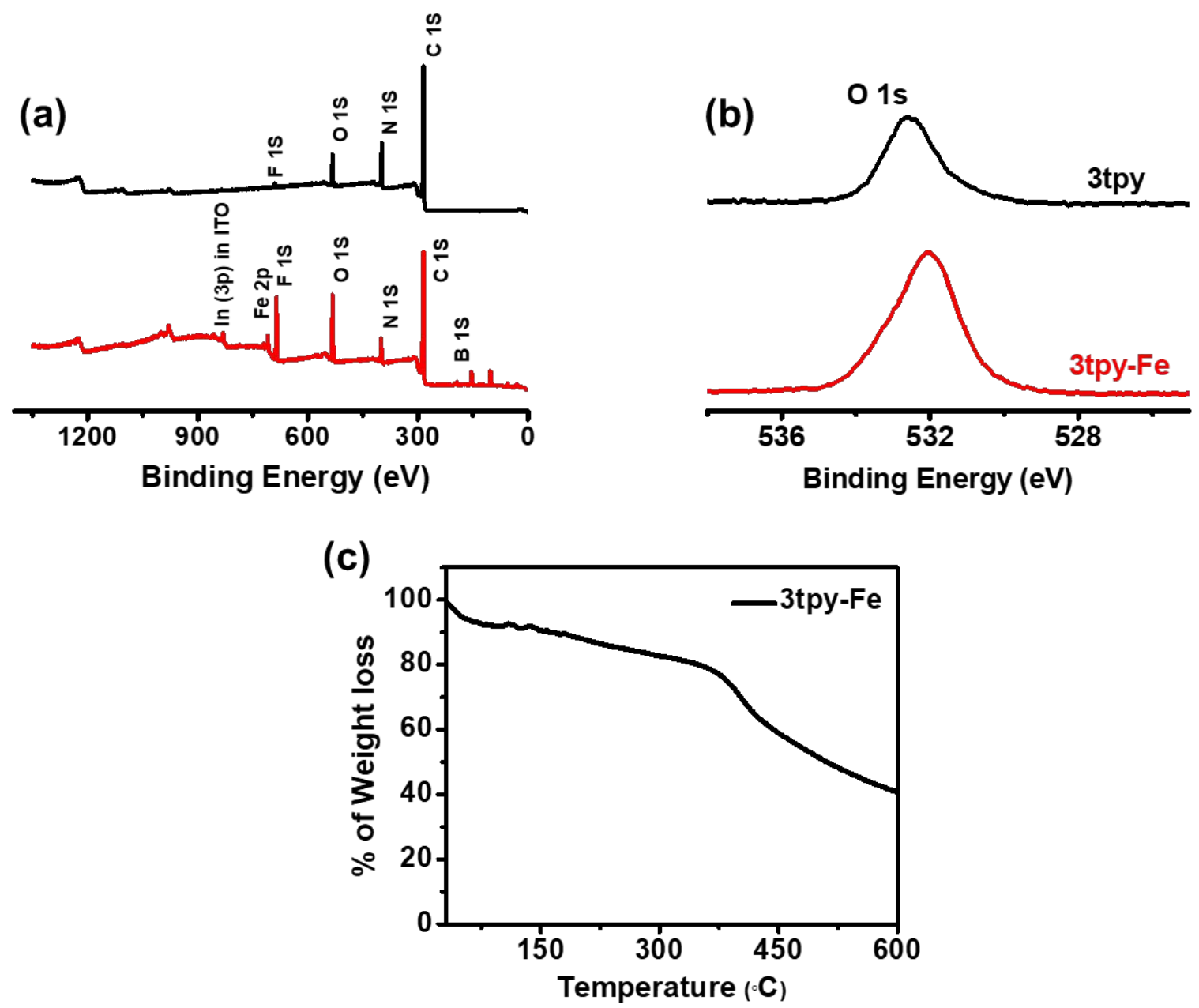

Figure S6. (a) XP survey spectra, (b) XP-spectra focusing on O 1s of 3tpy (black) and 3tpy-Fe (red). (c) Thermogravimetric analysis of 3 tpy-Fe in $\mathrm{N}_{2}$ environment. 
7. UV-Vis spectra comparison of 3tpy-Fe polymers prepared in bulk and interfacial polymerizations.

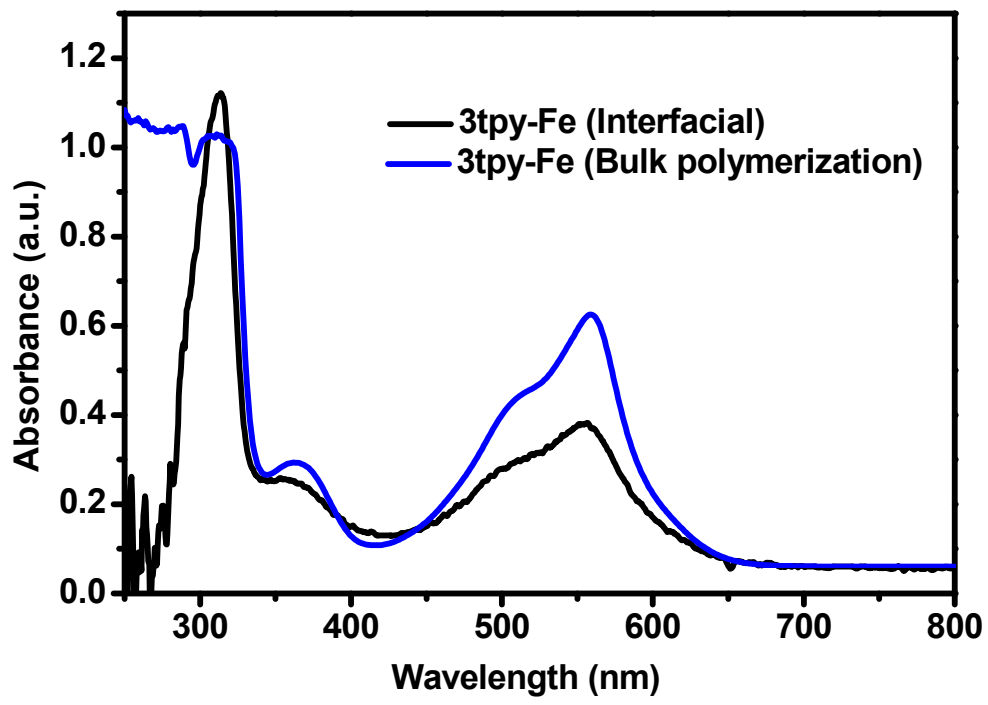

Figure S7. UV-Vis spectra comparison of 3tpy-Fe polymers prepared in bulk and interfacial polymerizations.

8. Peak current vs. scan rate study of 3 tpy-Fe film in three electrode system

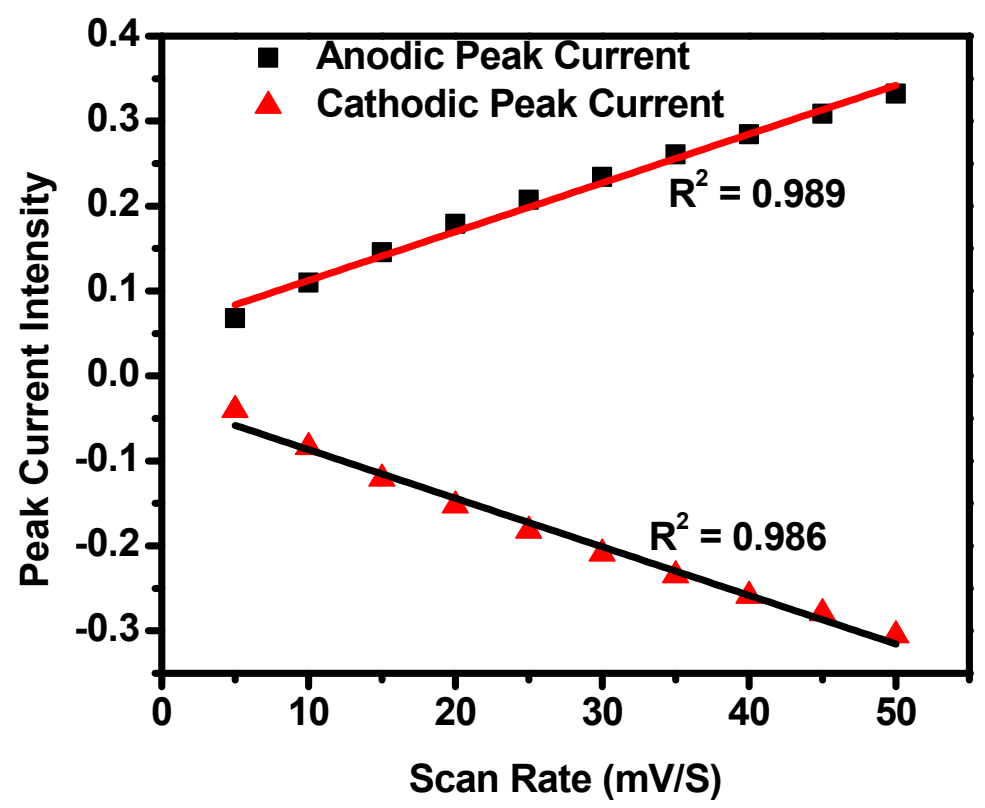

Figure S8. The linear correlations between the peak current and the scan rate during the redox cycle. The above one is for oxidation and below one for reduction and the corresponding $\mathrm{R}^{2}$ values for fitting are given. 
9. The EC properties of 3tpy-Fe film $(350 \mathrm{~nm})$ over ITO in three electrode system
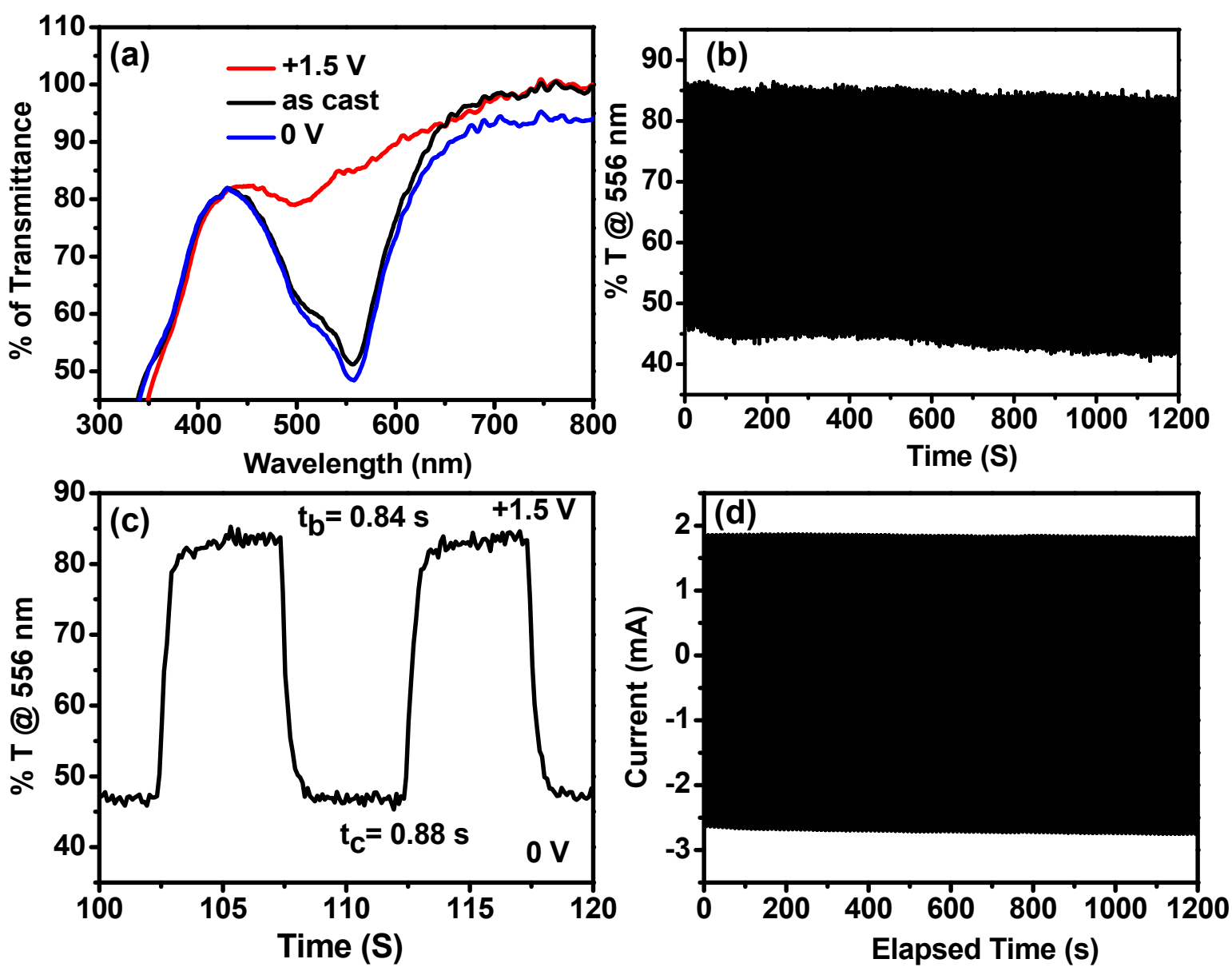

Figure S9. (a) Transmittance spectra of 3tpy-Fe film in three electrode system EC measurement by dipping the film in electrolyte solution. (b) Pulse switching stability ( $\Delta T$ vs time) of 3tpy-Fe film at $556 \mathrm{~nm}$ in $5 \mathrm{~s}$ interval time between $+1.5 \mathrm{~V}$ and $0 \mathrm{~V}$. (c) Response time for bleaching and coloration of 3tpy-Fe film, and (d) Chronoamperogram ( $i$ vs time) during spectrochemical switching time measurement. 
10. Electrochromic and chronoamperometry study of 3tpy-Fe film based ECD
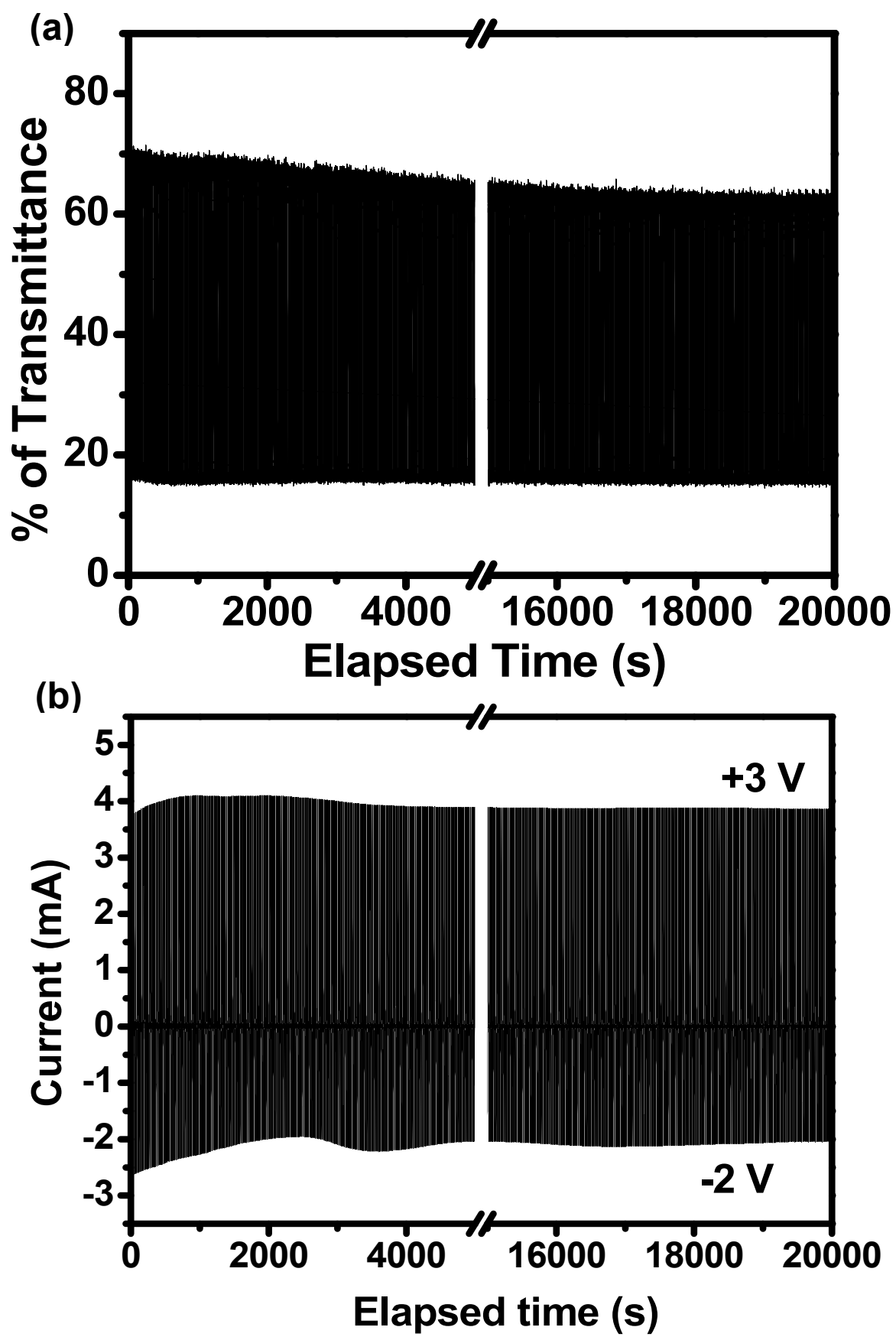

Figure S10. (a) Pulse switching cycle stability ( $\triangle T$ vs. time) of 3tpy-Fe based ECD at $556 \mathrm{~nm}$ up to 1000 cycles in 10 s interval time between $+3 \mathrm{~V}$ and $-2 \mathrm{~V}$. (b) Chronoamperogram ( $i$ vs. time) during spectro-electrochemical switching. 
Table S2: Comparison of electrochromic properties with other reported ECMs

\begin{tabular}{|c|c|c|c|c|c|c|c|}
\hline $\begin{array}{l}\text { Types } \\
\text { materials }\end{array}$ & $\begin{array}{l}\text { Molecular } \\
\text { unit/Framewo } \\
\text { rk/ binding } \\
\text { ligand }\end{array}$ & $\begin{array}{l}\text { Optical } \\
\text { contrast/ } \\
\text { transmittance } \\
\text { change }(\Delta T \%)\end{array}$ & $\begin{array}{l}\text { Coloration } \\
\text { efficiency } \\
\left(\eta \mathrm{cm}^{2} C^{-1}\right)\end{array}$ & $\begin{array}{l}\text { Solid state } \\
\text { ECD } \\
\text { fabrication }\end{array}$ & $\begin{array}{l}\text { Durability } \\
\text { in solid } \\
\text { state ECD }\end{array}$ & $\begin{array}{l}\text { Memory } \\
\text { study }\end{array}$ & Reference \\
\hline $\begin{array}{l}\text { Metallo- } \\
\text { supramolecular } \\
\text { polymer (MSP) }\end{array}$ & $\begin{array}{l}\text { Terpyridine } \\
\text { based ligand } \\
\text { metal complex }\end{array}$ & $\begin{array}{l}\text { Not reported as } \\
\text { done with } \\
\text { respect to Abs. } \\
\text { change }\end{array}$ & $\begin{array}{l}\text { Not } \\
\text { reported }\end{array}$ & Not reported & $\begin{array}{l}\text { Not } \\
\text { reported }\end{array}$ & $\begin{array}{l}\text { Close to } \\
15 \mathrm{~min}\end{array}$ & $\begin{array}{ll}\text { J. Am. } & \text { Chem. } \\
\text { Soc. } & \mathbf{2 0 0 8 ,} \\
130, & 2073- \\
2081 & \\
\end{array}$ \\
\hline $\begin{array}{l}\text { Metallo- } \\
\text { supramolecular } \\
\text { polymer (MSP) }\end{array}$ & $\begin{array}{l}\text { Terpyridine } \\
\text { based ligand }\end{array}$ & $\begin{array}{l}41.6 \text { (for } 1 D) \\
\text { and } 50.7 \text { for } 3 D \\
\text { polymers }\end{array}$ & $\begin{array}{lr}263.8 & \text { for } \\
1 \mathrm{D} & \text { and } \\
383.4 & \text { for } \\
\text { 3D } & \end{array}$ & Not reported & $\begin{array}{l}\text { Up to } 50 \\
\text { cycles }\end{array}$ & Not done & $\begin{array}{l}\text { ACS Appl. } \\
\text { Mater. } \\
\text { Interfaces } \\
\mathbf{2 0 1 4 ,} 6,9118- \\
9125\end{array}$ \\
\hline $\begin{array}{l}\text { Metal organic } \\
\text { molecular } \\
\text { assembly }\end{array}$ & $\begin{array}{l}\text { Polypyridyl } \\
\text { complex }\end{array}$ & 65 & 474 & Reported & $\begin{array}{l}\text { Up to } 75 \\
\text { cycles }\end{array}$ & Not done & $\begin{array}{lr}\text { J. Am. } & \text { Chem. } \\
\text { Soc. } & \mathbf{2 0 1 7}, \\
139, & 11471- \\
11481 & \end{array}$ \\
\hline $\begin{array}{l}\text { MSP- } \\
\text { nanocomposite }\end{array}$ & $\begin{array}{l}\text { Terpyridine } \\
\text { based polyFe- } \\
\text { nanoclay } \\
\text { composite }\end{array}$ & 62.8 & 572.77 & Reported & $\begin{array}{l}\text { Up to } 1000 \\
\text { cycles }\end{array}$ & Not done & $\begin{array}{lr}\text { Sol. } & \text { Energy } \\
\text { Mater. } & \text { Sol. } \\
\text { Cells } & \mathbf{2 0 2 0}, \\
208,110392 .\end{array}$ \\
\hline Inorganic oxide & $\mathrm{WO}_{3}$ & 97.7 & 118.3 & Reported & $\begin{array}{l}\text { Up to } 10 \\
\text { cycles }\end{array}$ & Not done & $\begin{array}{l}\text { Chem. Sci. } \\
\text { 2016, 7, 1373- } \\
\text { 1382 }\end{array}$ \\
\hline Pasmonic & $\begin{array}{l}\mathrm{Au} \text { and } \mathrm{Al} \\
\text { metallic } \\
\text { nanoslit arrays } \\
\text { on polyaniline }\end{array}$ & 90 & $\begin{array}{l}\text { Not } \\
\text { reported }\end{array}$ & Not reported & $\begin{array}{l}\text { Not } \\
\text { reported }\end{array}$ & Not done & $\begin{array}{l}\text { Nat. Commun. } \\
\text { 2016, 7, } 10479\end{array}$ \\
\hline $\begin{array}{l}\pi \text {-conjugated } \\
\text { polymer }\end{array}$ & $\begin{array}{l}\text { dioxythiophen } \\
\text { e }\end{array}$ & 72 & 375 & Not reported & $\begin{array}{l}\text { Not } \\
\text { reported }\end{array}$ & Not done & $\begin{array}{l}\text { Chem. Mater. } \\
\text { 2002, } 14 \text {, } \\
3964-3970\end{array}$ \\
\hline $\begin{array}{l}\text { Electropolymeri } \\
\text { zed film of } \\
\text { metal-complex }\end{array}$ & $\begin{array}{l}\text { biscyclometala } \\
\text { ted ruthenium } \\
\text { complex }\end{array}$ & 40 & 250 & Not reported & $\begin{array}{l}\text { Not } \\
\text { reported }\end{array}$ & $\begin{array}{l}\text { Memory } \\
\text { close to } \\
1 \mathrm{~h}\end{array}$ & $\begin{array}{lr}\text { J. Am. } & \text { Chem. } \\
\text { Soc. } & \mathbf{2 0 1 1} \text {, } \\
133, & 20720- \\
20723 & \end{array}$ \\
\hline CONASHs & $\begin{array}{l}\text { Terpyridine- } \\
\text { metal complex }\end{array}$ & $\begin{array}{l}\text { Not reported as } \\
\text { done with } \\
\text { respect to Abs. } \\
\text { change }\end{array}$ & $\begin{array}{l}\text { Not } \\
\text { reported }\end{array}$ & Reported & $\begin{array}{l}\text { Up to } 5 \\
\text { cycles }\end{array}$ & Not done & $\begin{array}{lr}\text { J. Am. } & \text { Chem. } \\
\text { Soc. } & \mathbf{2 0 1 5} \text {, } \\
137, & 4681- \\
4689 & \end{array}$ \\
\hline CONASHs & $\begin{array}{l}\operatorname{Bis}(2,2 '- \\
\text { bipyridine }) \\
\text { based ligand }\end{array}$ & 62 & 431 & shown & $\begin{array}{l}\text { Up to } 300 \\
\text { cycles }\end{array}$ & Not done & $\begin{array}{lr}\text { ACS } & \text { Appl. } \\
\text { Mater. } & \\
\text { Interfaces } \\
\text { 2019, 11, } \\
11893-11903\end{array}$ \\
\hline CONASH & $\begin{array}{l}\text { Terpyridine- } \\
\text { metal } \\
\text { complex with } \\
\text { non- } \\
\text { conjugated } \\
\text { ligand }\end{array}$ & 53.19 & 470.16 & Shown & $\begin{array}{l}\text { Up to } 1000 \\
\text { cycles }\end{array}$ & $\begin{array}{l}50 \% \\
\text { retentio } \\
n \text { in } 25 \\
\text { min }\end{array}$ & This work \\
\hline
\end{tabular}


11. EC memory as a function of thickness of 3tpy-Fe film

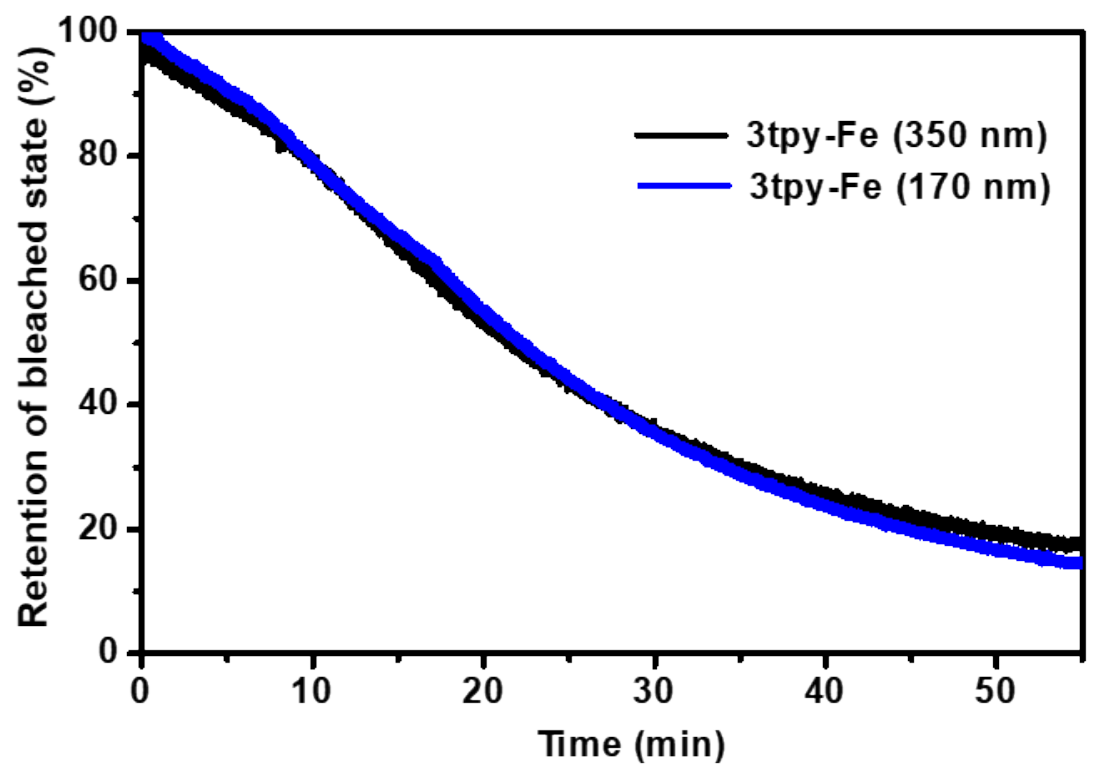

Figure S11. Transmittance decay (normalized electrochromic memory) curve of 3tpy-Fe films with different thickness from bleached state to colored state at open circuit condition.

12. Thickness measurement of polyFe and 3tpyC-Fe film
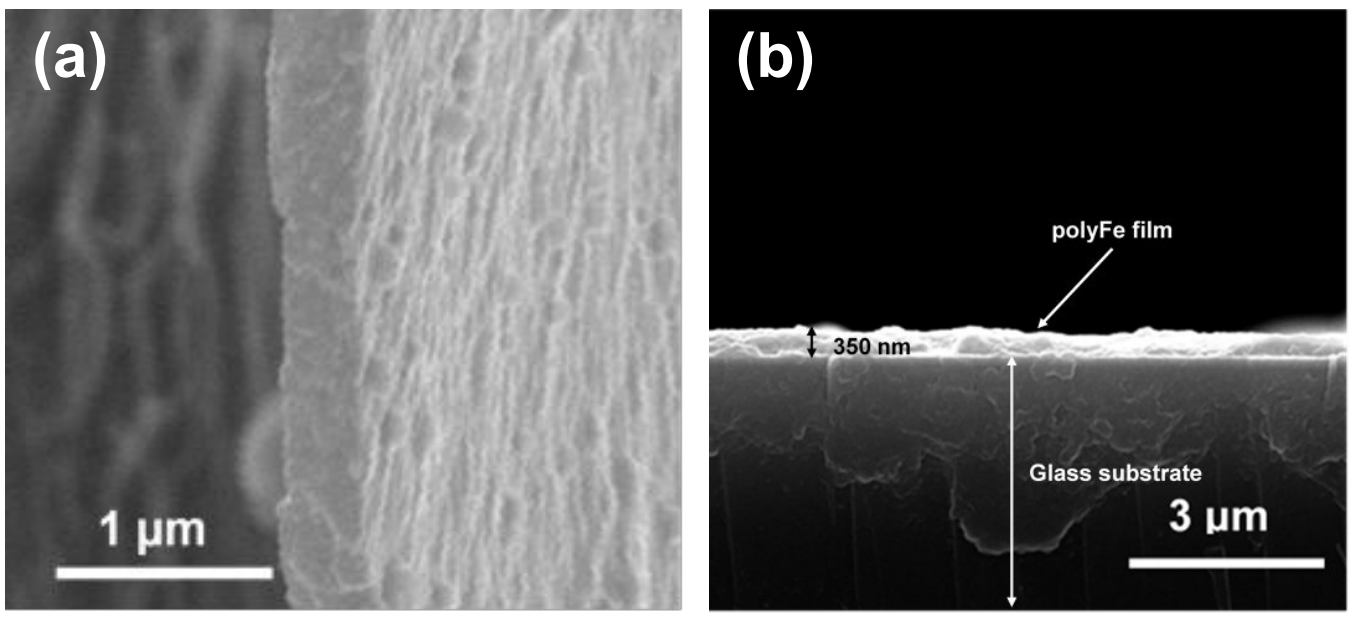

Figure S12. Thickness measurement through cross-sectional FESEM study of (a) 3tpyC-Fe and (b) polyFe film over a glass substrate. The average thickness of the film was measured to be around $350 \mathrm{~nm}$. 